\title{
Materials \& Design Virtual Special Issue Editorial: Analysis of material microstructure and defects using X-ray and neutron beams - Size-Strain
}

Ian K. Robinson, Alexander M. Korsunsky and Tan Sui

Beams of penetrating radiation, such as X-ray photons, neutrons and electrons, provide the basis for a whole host of tools for probing material structure, composition and properties. In particular, over a century of using reciprocal space methods based on diffraction has allowed revealing intricate details of complex molecules, collecting multi-scale information about different levels of structural organization, observing the course of reactions and processes, etc.

Although methods and approaches in this field are well-established, the proliferation of hardware and software tools in the recent years has led to a considerable increase in the ease of access to many techniques. This increases their attractiveness for the community, and an ever greater number of researchers make use of large scale facilities and advanced instruments.

The 7th Size-Strain conference 'Diffraction Analysis of the Microstructure of Materials' (SS-VII) was held at Oxford from 21 to 24 September 2015. The 80 participants were able to enjoy four days of college living at Trinity College, whilst all plenary conference sessions were held at the nearby Department of Engineering Science of the University of Oxford. On the final day of the conference, a half-day trip was arranged to the large scale facilities at the nearby Harwell campus, Diamond Light Source (DLS), the UK synchrotron, and ISIS neutron spallation source. The conference planning, booking and logistics were run extremely smoothly and efficiently by the DLS events team. The meeting was co- chaired by Ian Robinson (University College London) and Alexander Korsunsky (University of Oxford). 
The scientific programme of the conference continued the themes set and addressed at the previous meetings in Garmisch-Partenkirchen (SS- V) and Giens (SS-VI). These address the investigation of material micro- structure and properties by diffraction methods, with a special interest in their application to polycrystalline materials, the methodologies for the study of lattice defects, residual stress and texture in thin films, nano-structures and at surfaces, line-broadening analysis, lineprofile fitting, and modelling based on the fundamental parameters. In addition to the new and by now well-established topics of microbeam diffraction and coherent diffraction introduced at SS-VI, the SS-VII saw the addition of X-ray and neutron imaging and Pair Distribution Function (PDF) analysis.

The conference was opened with an inspiring plenary talk by Cev Noyan (Columbia Uuniversity) entitled "The Anatomy of a Powder Diffraction Experiment", which prompted a lively discussion and set the mood for open and active exchange of views, experiences and ideas. Further invited presentations were given by Clare Grey (Cambridge) on "X-ray Diffraction Methods for Studying Structure and Dynamics in Batteries and Supercapacitors", Thomas Hansen (ILL) on "Stacking faults in ice", Felix Hofmann (Oxford) on "Probing Atomic Scale Defects", Alberto Leonardi (Indiana) on "Microstrain in nanomaterials: XRD line profile interpretation enhanced by Molecular Dynamics simulations",

Reinhard Neder (Erlangen) on "Size and strain analysis of small highly disordered nanoparticles" and by Tobias Schulli (ESRF) on "The nanodiffraction Beamline ID01/ESRF: diffraction microscopy and coherent diffraction imaging for high resolution structural analysis". Instead of split- ting the conference into parallel sessions, the chairs took the decision to treat all participants to the opportunity to obtain a complete view of the research landscape in the field by attending all focused sessions on the key topics, including Disorder, Defects, Morphology, Nanoscience, Thin Films, Alloys, Oxides, Strain Mapping, Dynamics, and 3D Imaging.

The Virtual Special Issue of Materials and Design captures some of the interesting work presented at the conference in the form of contributed full length articles. 
Particular care has been taken by the editorial team to ensure that the publications were in line with the journal scope and editorial preferences described in the editorial note [1]. This Virtual Special Issue demonstrated the wide variety of applications of advanced diffraction methodologies in modern materials science. The full details of published articles can be found at the VSI web page:

Acknowledgements

The Special Issue Guest Editors express sincere appreciation to all authors and reviewers for their dedication in putting together a high quality body of joint work. Our gratitude is also due to the Materials \& Design editorial team and technical staff for their cooperation and excel- lent service. Financial support from Diamond Light Source towards running Size-Strain VII is gratefully acknowledged, as are the contributions to event organization from Zoe Cattell and Sarah Bucknall (DLS), and Eva Williams (Engineering Science). Particular thanks are due to Kevin Knott CVO, Estates Bursar of Trinity College, for the permission to use college facilities.

\section{References}

[1] A.M. Korsunsky, A.G. Gibson, G.D. Nguyen, M. Sebastiani, X. Song, T. Sui, Editorial note - on the aims \& scope and priority areas in Materials \& Design, Mater. Des. 88 (2015) 1377-1380.

Ian Robinson is group leader in the Condensed Matter Physics and Materials Science De- partment at Brookhaven National Laboratory, and Professor at the London Centre for Nanotechnology, University College. His research is currently focused on the development of coherent X-ray diffraction methods for image the structure of nanoparticles. His re- search makes extensive use of synchrotron radiation and Free Electron Lasers. He built a beamline at Brookhaven to develop Surface X-ray Diffraction and a second one at Argonne for Coherent X-ray Diffraction. One outcome of the work was the discovery of Crystal Truncation Rods, for which he was awarded the Surface Structure Prize in 2011 and the Gregori Aminoff Prize in 2015. 
Alexander M. Korsunsky, Professor of Engineering Science in the University of Oxford, and Head of the Oxford MBLEM lab, is also Editor-in-Chief of Materials \& Design. He is a world-leading expert in the analysis of deformation, structure and function of materials across the scales.

Tan Sui conducts research in the field of functional and structural properties of advanced materials and devices, from natural tissues to electronics systems, making extensive use of synchrotron X-rays for imaging, diffraction and spectroscopy studies. She is in charge of advanced instrumentation for multi-modal microscopy at the Oxford MBLEM lab, and is also Managing Editor of Materials \& Design.

Ian K. Robinson

Condensed Matter Physics and Materials Science Department, Brookhaven National Laboratory, Upton NY 11973, USA London Centre for Nanotechnology, University College, London, UK Corresponding author. E-mail address: i.robinson@ucl.ac.uk.

Alexander M. Korsunsky 